\title{
Efectividad del i_map en la prevención de plagio académico a nivel universitario
}

\section{Effectiveness of i_map in preventing aca- demic plagiarism at a college level}

\author{
Maryory Medina Turcios* \\ mmedinat@upnfm.edu.hn \\ César Leonardo Alvarado Alvarado** \\ calvarado@upnfm.edu.hn
}

\section{Resumen}

El plagio académico es una práctica común entre estudiantes universitarios. Tradicionalmente esta temática ha sido abordada a través de estudios que determinan los factores generadores de la misma, sin ahondar en estrategias efectivas que prevengan dichas prácticas. Por tanto, para este estudio se utilizó una estrategia novedosa llamada i_map, que reduce el plagio académico a través del fortalecimiento de las habilidades de búsqueda y manejo de información, documentación del proceso de investigación y generación de ideas propias por parte del estudiante.

El objetivo de este estudio es determinar la efectividad del i_map en la prevención del plagio académico y compararla con estrategias tradicionales como el reforzamiento en el uso de paráfrasis y citas textuales. La investigación fue desarrollada en tres espacios pedagógicos de la Carrera de Ciencias Naturales del

* Docente del Departamento de Ciencias Naturales, Centro Universitario de Educación a Distancia, Universidad Pedagógica Nacional Francisco Morazán.

** Docente del Departamento de Matemáticas, Universidad Pedagógica Nacional Francisco Morazán.

Recibido 24 de agosto 2017 /Aceptado 30 de octubre 2017. 
CUED-UPNFM, cada espacio fue diferenciado según el tipo de estrategia utilizada (i_map, talleres de paráfrasis y citas textuales, equipo control) para la elaboración de ensayos académicos. En total fueron analizados 74 ensayos. El análisis de los datos fue realizado a través de un ANOVA de factor único y Welch test. Los niveles más bajos de plagio $(p=0.0003246)$ y los valores medios más altos de fuentes académicas citadas $(p=0.0100)$ fueron registrados en los trabajos de los estudiantes que utilizaron el i_map. Este estudio concluye la efectividad, estadísticamente significativa, del uso del i_map en la prevención de plagio académico, por lo que sugerimos la inclusión de esta estrategia como herramienta para prevenir dicha práctica y a la vez mejorar la redacción de asignaciones escritas.

Palabras clave: i_map, plagio, ensayo, paráfrasis, citas.

\section{Abstract}

Academic plagiarism is a common and widespread practice among university students. Traditionally, this topic has been approached through studies that determine the factors that cause such practice, without delving into effective strategies to prevent it. Therefore, for this study we used a novel strategy called i_map, that reduces academic plagiarism through the strengthening of the search and information management skills, documentation of the research process and generation of own ideas by the student.
The objective of this study was to determine and compare the effectiveness of i_map in the prevention of academic plagiarism with traditional strategies such as the reinforcement in paraphrasing and quoted text. The research was developed in three pedagogical spaces of the natural sciences career of CUED-UPNFM, each space was differentiated according to the type of strategy used (i_map, workshops of paraphrasing and textual citations, control team) for the development of academic essays. A total of 74 essays 
were analyzed. Data analysis was performed using a single-factor ANOVA and Welch test. The lowest levels of plagiarism ( $p=$ $0.0003246)$ and the highest mean values of cited academic sources $(p=0.0100)$ were recorded in the works of students who used i_map. This study concludes the effectiveness, statistically significant, of the use of i_map in the prevention of academic plagiarism; thus, we suggest the inclusion of this strategy as a tool to prevent such practice and at the same time to improve written assignments.

Keywords: i_map, plagiarism, essays, paraphrasing, citation.

\section{Introducción}

Evitar el plagio académico es uno de los grandes desafíos que afrontan los estudiantes y los centros de educación superior. Esta práctica deshonesta ha surgido como una seria problemática en más de alguno de nuestros espacios pedagógicos, siendo común escuchar entre los docentes la severidad en los niveles de plagio de sus estudiantes. Esto ha generado una preocupación entre la comunidad docente en relación con las estrategias de investigación, redacción de informes y ensayos académicos que requieren el uso de información de otros autores. Con ello, algunas universidades han justificado el establecimiento de políticas que regulen y castiguen a los estudiantes que cometen plagio, sin embargo, es sabido que estos niveles de plagio están directamente asociados a la severidad de las sanciones establecidas por las instituciones (Bennett, 2005).

Siendo esta una problemática latente, numerosos son los estudios que han documentado las posibles causas del plagio a nivel universitario, siendo relativamente pocos los que enfatizan algunas estrategias que tienen como fin la prevención del mismo (Gullifer y Tyson, 2010; Walden y Peacock, 2006). Lo antes expuesto, fue el origen de este estudio, donde se buscaron estrategias para disminuir los niveles de plagio de 
los estudiantes. Dentro de los estudios previos relacionados a esta temática, sobresale la investigación realizada por Walden y Peacock (2006), docentes de la Universidad de Hertfordshire en el Reino Unido, quienes diseñaron una estrategia llamada i_map (forma corta de mapa para el manejo de la información por su significado en inglés) con un enfoque útil en la prevención del plagio académico.

El i_map es el término que describe el "proceso estructurado que requiere a los estudiantes hacer un seguimiento de sus actividades de investigación, un registro de los procesos y actividades de pensamiento, mientras participan en la recopilación, evaluación, selección y presentación de información extraída de diversas fuentes" (Walden y Peacock, 2008, p.133). Esta estrategia fue concebida para fortalecer las habilidades de búsqueda y manejo de la información, documentando el proceso de investigación de una temática en particular, las ideas principales y aportes de los estudiantes sobre la información recopilada a través de una serie de pasos (ver discusión teórica). El i_map requiere que el estudiante no solo recopile información, sino que razone sobre la misma y genere ideas a partir de ella, previo a la elaboración o redacción de asignaciones escritas.

El i_map también es una herramienta de comunicación visual, dado que los procesos involucrados en la elaboración del mismo pueden ser representados gráficamente (Figura 1). Esta herramienta es muy útil para estudiantes y docentes, ya que estos pueden utilizarla como una guía para la elaboración de escritos, y en el caso del docente puede utilizarla para efectos de evaluación y retroalimentación de los procesos de investigación (Walden y Peacock, 2006). Estudios posteriores a los de Walden y Peacock (2006) han explorado los múltiples beneficios de esta estrategia, dentro de los cuales sobresalen sus aportes en la prevención de plagio académico (Emerson, Stevens y Muirh, 2008). Para efectos de este estudio, la investigación se centró únicamente en el beneficio del i_map para la prevención del plagio académico. 
Otra estrategia que contribuye en la disminución de los niveles de plagio es el reforzamiento en las habilidades de paráfrasis (Walker, 2008). Por ejemplo, Holt (2012) en su investigación con estudiantes de Ecología general, registró una mejora en las habilidades de detección y prevención del plagio académico mediante el entrenamiento en paráfrasis. Para efectos de esta investigación y con base en los resultados antes descritos, se brindó reforzamiento en paráfrasis y citas textuales bajo la normativa APA (American Psychological Association, 2010), este conjunto de actividades fue denominado talleres de paráfrasis y citas textuales.

El objetivo de esta investigación es determinar la efectividad del i_map en la disminución del plagio académico y compararla con los talleres de paráfrasis y citas textuales. La razón por la cual dichas estrategias fueron contrastadas es porque ambas tienen entre sus beneficios la prevención del plagio académico, además el i_map es una estrategia novedosa con múltiples ventajas en relación con los talleres de paráfrasis y citas. Se espera registrar bajos niveles de plagio en las asignaciones de los estudiantes que utilizaron el i_map en comparación a la otra estrategia utilizada.

El estudio fue desarrollado con tres espacios pedagógicos de la Carrera de Ciencias Naturales del Centro Universitario de Educación a Distancia (CUED) de la Universidad Pedagógica Nacional Francisco Morazán (UPNFM). En este artículo se presentan los resultados de un diagnóstico efectuado a dichos estudiantes, con lo que se corroboró, entre otros aspectos, el poco conocimiento sobre temáticas de plagio. Este estudio fue desarrollado en dos etapas, en la primera de ellas se verificó los niveles de plagio en las asignaciones de los estudiantes, sin la influencia de alguna estrategia para la prevención del plagio. En la segunda etapa los espacios pedagógicos fueron diferenciados según el tipo de estrategia utilizada, con el fin de determinar la efectividad de las mismas en la reducción del plagio académico. 


\section{Discusión Teórica}

El plagio académico es una práctica común entre estudiantes universitarios, con la digitalización de la información dicha práctica ha incrementado exponencialmente, ya que los estudiantes no cuentan con las habilidades necesarias para manejar la información disponible en línea (Fintan y Lancaster, 2001). Plagio "es la apropiación de las ideas, procesos, resultados o palabras de otra persona sin dar crédito apropiado" (Office of Science and Technology Policy, 2000, "Federal Policy on Research Misconduct," párr. 7). Algunos autores consideran que la permisión de este tipo de plagio fomenta la corrupción no solo en el ámbito académico (Diez-Martínez, 2014), siendo esta una de las razones principales por las que se debe dar seguimiento detallado a este tipo de prácticas presentes en diferentes áreas académicas. A pesar de ser esta una conducta de ninguna manera justificable es necesario mencionar que algunos autores atribuyen la ocurrencia de estas prácticas al desconocimiento de las acciones que implican el plagio (Voelker, Love y Pentina, 2011). Considerando los factores generadores del plagio, Beasley (2004) establece algunos tipos de plagio ligados al conocimiento y actitudes de los estudiantes frente a esta práctica, siendo estos:

Plagio accidental: el estudiante no entiende la definición de plagio o comete un error al citar o parafrasear. Es ocasionado por la ignorancia, exceso de información y organización.

Plagio oportunista: el estudiante sabe que es incorrecto plagiar, pero de igual forma lo hace debido a la desorganización, exceso de la información, fallos en la conducta ética, pereza o miedo (p. 9).

Así, el plagio no siempre es accidental, sin embargo, las sanciones establecidas para esta práctica no distinguen entre los tipos de plagio o razones por las que el estudiante incurre en él. En cualquiera de los casos, es necesario establecer las acciones por las que un estudiante puede incurrir en plagio, siendo estas: 
Convertir el trabajo de otra persona como propio, copiar palabras o ideas de otra persona sin dar crédito, no colocar una cita entre comillas, brindar información incorrecta sobre la fuente de una cita, cambiar las palabras de la oración por sinónimos y mantener la estructura de la misma sin dar crédito y copiar la mayoría de palabras o ideas de una fuente ya sea que se otorgue o no crédito (Plagiarism, 2017, párr. 4).

Las sanciones relacionadas al plagio académico varían y dependen de la institución educativa, estas pueden incluir llamados de atención, no valoración de las asignaciones y en algunos casos la suspensión de la asignatura (Berlinck, 2011). Dichas acciones son necesarias para sentar precedentes ante esta práctica, sin embargo, tal como lo establece Walden y Peacock (2006), es más importante aún establecer porque es necesario evitar el plagio antes que sus sanciones. Así, el plagio tiene profundas consecuencias en la educación superior, en la valoración del conocimiento de otros autores y en los conceptos de propiedad intelectual (Walden y Peacock, 2008). Es decir, esta práctica deshonesta ha debilitado el uso de algunas actividades de aprendizaje como el ensayo académico, ya que generalmente los estudiantes cometen plagio, obligando al docente a prescindir de este tipo de asignaciones por no cumplir con el objetivo final de este.

Valorando los procesos preventivos antes que los de castigo, algunos autores han medido la influencia de algunas estrategias en la prevención del plagio. Tal es el caso de Holt (2012) y Walker (2008) quienes invirtieron esfuerzos en documentar los efectos de reforzar las habilidades de paráfrasis en la prevención del plagio. Paráfrasis "es el acto de poner un pasaje del material fuente en palabras propias atribuyéndolo a la fuente original" (Online Writing Lab at Purdue University, 2013, párr.4). Dichos estudios han mostrado resultados eficientes al registrar un mejor desempeño en la paráfrasis de textos de diferentes grados de dificultad (Holt, 2012; Walker, 2008).

Sin embargo, es evidente que la prevención de esta práctica deshonesta va más allá del mejoramiento en las habilidades de interpretación de la información. Es decir, se necesita estrategias holísticas que permitan 
abordar esta problemática en un contexto altamente digitalizado. Un ejemplo de este tipo de estrategia es el i_map, desarrollado por K. Walden y A. Peacock (2006), docentes de la Universidad de Hertfordshire, Reino Unido, quienes después de haber experimentado durante tres años con esta estrategia, documentaron su experiencia en el artículo titulado "The i_map: a process-centered response to plagiarism". Según Walden y Peacock (2006) el i_map es una herramienta que facilita la visualización y el análisis de la información de diversas fuentes. Dentro de sus múltiples beneficios se destacan el aporte al proceso de estructuración de una opinión escrita y la prevención del plagio académico (Emerson, Stevens y Muirh, 2008).

Para elaborar un i_map deben ser consideradas las siguientes fases:

1. Objetivo de la actividad: el estudiante debe definir el propósito de la asignación, en este apartado se debe incluir la audiencia y el formato del mismo. Se definirá el tema del ensayo, las variables que intervienen y la delimitación del mismo.

2. Alcance de la información: el estudiante establece los temas y la información que necesita encontrar. Para ello formulará una serie de preguntas relacionadas a la temática del ensayo, las que posteriormente responderá en el mismo.

3. Estrategias de recolección de información: incluye fuentes electrónicas o impresas, nombres de bases de datos a ser utilizadas, motores de búsqueda en internet. Esta lista debe ser muy objetiva.

4. Evaluación del material (información) colectada: lectura analítica de la información colectada y formación de un argumento a través de las ideas exploradas.

5. Edición y selección del material: selección de párrafos claves que podrían ser utilizados como citas para dar soporte a nuestro argumento.

6. Construcción de un texto preciso: aquí expresará en ideas o palabras propias los componentes de la asignación.

7. Borrador final: finalización del texto, aquí deberá hacer una verificación del vocabulario utilizado, de la claridad de las ideas 
que expresa, verificación de las citas y bibliografía utilizada (Walden y Peacock, 2006, p. 206).

Posteriormente, los resultados de estas fases son representados en un mapa que puede incluir diferentes elementos como imágenes que sustituyan una palabra clave, gráficos que simbolicen información valiosa para los estudiantes, direcciones electrónicas que recuerden sitios importantes de donde pueden obtener información, palabras clave, entre otros elementos que pueden variar dependiendo de las necesidades o estilos de aprendizaje de cada estudiante. Así, no existe un formato para representar las fases del i_map, haciéndolo aún más valioso, considerando que el aprendizaje es un proceso individual (Figura 1).

\section{Métodos y materiales}

El estudio fue desarrollado durante el segundo período académico del 2017 en tres espacios pedagógicos, Ecología (sección A y B) y Seminario de Historia Natural, de la Carrera de Ciencias Naturales de la UPNFM-CUED. En los tres espacios pedagógicos fue aplicado un diagnóstico con preguntas abiertas y cerradas que tenían como objetivo colectar información relacionada a los conocimientos previos y percepción de los estudiantes sobre plagio académico.

El interés principal de este estudio fue evaluar el desempeño del i_map en la disminución de los niveles de plagio académico y compararlo con los talleres de paráfrasis y citas textuales, que incluía una serie de actividades orientadas a la identificación y construcción de citas textuales y de paráfrasis, uso adecuado de la normativa APA para dar crédito a los autores y construcción de listas de referencias bajo dicha normativa. Así, únicamente la sección $B$ de Ecología utilizó el i_map y la sección $A$, solo los talleres de paráfrasis y citas textuales. Los estudiantes de Seminario de Historia Natural sirvieron como equipo control, quienes no recibieron ningún tipo de reforzamiento en la prevención del plagio. 
Uso de las estrategias con los estudiantes

El i_map y los talleres de paráfrasis y citas textuales fueron introducidos en el segundo encuentro del periodo académico en cuestión. La introducción de dichas estrategias inició con una reflexión sobre la importancia de evitar el plagio académico y las consecuencias de este. El tiempo empleado para desarrollar cada estrategia fue el mismo (20-30 minutos) con cada espacio pedagógico.

i_map: inicialmente fue establecido el concepto de plagio académico, posteriormente la definición del i_map y las fases para su elaboración. Cada estudiante recibió un resumen textual con información del i_map basada en el estudio de Walden y Peacock (2006). También fueron mostrados y discutidos algunos ejemplos de i_map. Finalmente fue establecido el tema sobre el cual los estudiantes realizarían una investigación y presentarían los resultados en un ensayo académico. En encuentros posteriores, los estudiantes hicieron una breve presentación en clase donde explicaron cada uno de los pasos del i_map elaborado (Figura 1); esta actividad fue realizada una semana antes de la entrega de los ensayos académicos.

Talleres de paráfrasis y citas textuales: estos talleres iniciaron con la introducción de los conceptos de plagio, paráfrasis, citas textuales y la forma en que estas deben presentarse bajo la normativa APA 6. Los estudiantes recibieron un manual resumen elaborado por el Centro de escritura Javeriano de la Pontificia Universidad Javeriana (2011) sobre la normativa APA 6, con ejemplos de citas textuales, de paráfrasis y una lista de ejemplos para referenciar diferentes fuentes. En cada taller los estudiantes construyeron citas textuales y de paráfrasis de artículos proporcionados por el docente. Particularmente, se enfatizó en la correcta paráfrasis abordando las diferencias con la paráfrasis mecánica, como establecido por Plagiarism (2017). 
Figura 1

¿_Map elaborado por los estudiantes de Ecología sección B

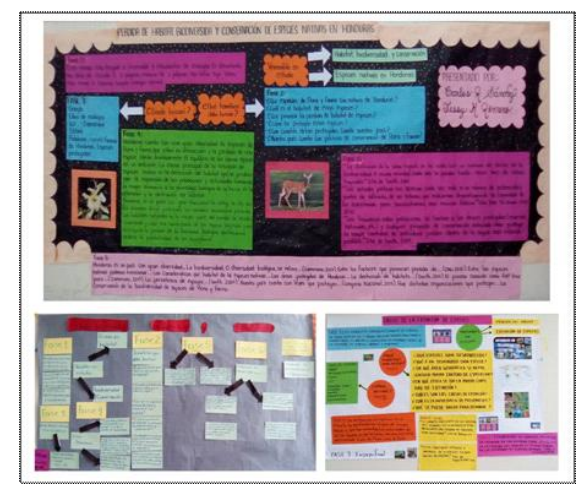

Nota: Algunos ejemplos de i_map realizados por los estudiantes, previo a la elaboración de los ensayos. Estos ejemplos muestran el uso de códigos de color para relacionar algunas fases, el uso de imágenes para representar términos claves y la variedad de formas en que son presentadas las fases del i_map.

\section{Criterios para establecer los niveles de plagio}

Los estudiantes de los tres espacios pedagógicos debían presentar tres ensayos durante el periodo académico. A través de estos ensayos los estudiantes podían prevenir el plagio mediante el uso de citas textuales o de paráfrasis y utilización correcta de la normativa APA 6 con el fin de dar crédito a los autores citados. Para establecer los niveles de plagio en los ensayos fueron utilizados los siguientes criterios:

Criterio 1 (C1): cantidad de citas correctas. Fueron consideradas como citas correctas únicamente las citas textuales o de paráfrasis libres de plagio, correctamente citadas y referenciadas bajo la normativa APA 6. Este criterio fue establecido basado en los resultados de estudios previos que indican que la mayoría de estudiantes creen evitar el plagio colocando únicamente el nombre del autor y año de la obra, obviando una correcta paráfrasis y uso correcto de comillas (Holt, 2012). Para hacer más objetiva la medición de este estudio únicamente fueron considerados los párrafos citados, ya que a través de la lista de 
referencias se pudo verificar la fuente y así objetivamente descartar la detección o no de plagio en los ensayos. También fue utilizado el software libre Plagiarisma (Plagiarisma, 2010) para corroborar la detección de plagio en la cita a la cual el estudiante hacía referencia. Criterio 2 (C2): tipo de fuente utilizada. Para ello fue clasificada y contabilizada la lista de referencias de cada ensayo (únicamente fueron consideradas las referencias de las citas correctas), utilizando una escala que incluía fuentes académicas (artículos científicos, libros, tesis) y no académicas (blogs de opinión, por ejemplo). Bajo las condiciones antes mencionados fueron contabilizados los dos criterios para cada ensayo presentado.

Cada ensayo fue revisado bajo la misma rúbrica de evaluación, siendo ésta de conocimiento previo de los estudiantes. Después de la presentación y revisión de cada ensayo, los estudiantes de los tres espacios pedagógicos recibieron comentarios de retroalimentación en relación con cada criterio de evaluación establecido en la rúbrica antes mencionada. Es necesario mencionar que fueron utilizados más criterios para la evaluación de los ensayos, sin embargo, para efectos del presente estudio únicamente fueron considerados los dos antes mencionados por ser de carácter cuantitativo facilitando así el análisis estadístico.

\section{Recolección de los datos}

La recolección de datos fue realizada en las etapas descritas a continuación: Etapa 1: durante el primer encuentro con los estudiantes fue solicitada la entrega del primer ensayo. Para los tres espacios pedagógicos fueron establecidos los mismos criterios de evaluación, tiempo de entrega (espacio de dos semanas para la entrega de cada ensayo) y no fue brindado ningún tipo de reforzamiento sobre cómo evitar el plagio académico, esto con el objetivo de establecer comparaciones posteriores entre los estudiantes que utilizaron el i_map con los que recibieron los talleres de paráfrasis y citas textuales y el equipo control. 
Etapa 2: en esta etapa los espacios pedagógicos fueron diferenciados según el tipo de estrategia utilizada por los estudiantes para disminuir los niveles de plagio en sus ensayos. Es decir, los estudiantes de una sección de Ecología recibieron información sobre cómo elaborar un i_map, mientras que los estudiantes de la otra sección de Ecología recibieron los talleres. Finalmente, el Seminario de Historia Natural sirvió como equipo control ya que no recibieron información sobre la prevención del plagio académico. Una vez brindada la información a cada espacio pedagógico, fue solicitada la entrega del segundo ensayo (con un tiempo máximo de entrega de dos semanas). Dos semanas después de haber entregado el segundo ensayo (bajo las mismas condiciones antes establecidas) fue solicitada la entrega del ensayo tres teniendo como tiempo máximo de entrega dos semanas.

\section{Análisis de los datos}

Pruebas de normalidad fueron realizadas en todos los conjuntos de datos. Para comparar el efecto del i_map con el equipo control y los talleres de paráfrasis y citas textuales en la disminución de los niveles de plagio académico, fue utilizado un análisis de varianza (ANOVA) de factor único $(\alpha=0.05)$, seguido de un test Tukey $(\alpha=0.05)$ en caso necesario (cuando los resultados de la ANOVA revelaban diferencias significativas entre los grupos en estudio fue utilizado el test Tukey para detectar específicamente el conjunto de datos que ocasionaba dicha variación). Dicho análisis fue realizado por separado para los ensayos de la Etapa 1 (ensayos entregados por los estudiantes de los tres espacios pedagógicos cuando estos no recibieron ningún tipo de entrenamiento sobre temáticas de plagio académico) y Etapa 2 (ensayos 2 y 3 que fueron elaborados después de haber brindado las indicaciones del i_map, talleres y el grupo control, respectivamente).

Los avances de los estudiantes que utilizaron el i_map fueron medidos a través de un t-test (Welch test, $\alpha=0.05$ ), lo que permitió hacer una comparación antes y después del uso de esta estrategia en relación con los niveles de plagio. Todos los análisis estadísticos fueron generados en el software estadístico R.

<Paradigma> - Revista de Investigación Educativa. Año 24. No. 38 


\section{Resultados}

\section{Conocimiento previo de los estudiantes}

Fueron encuestados 51 estudiantes de la Carrera de Ciencias Naturales (CUED-UPNFM). Algunos resultados de este diagnóstico son presentados en la Figura 2. Estos, reflejan que la mayoría de los estudiantes conoce y define correctamente el término plagio académico (76\%), sin embargo, el $67 \%$ de los encuestados admitió desconocer la definición de paráfrasis.

A pesar de que los estudiantes perciben el plagio académico como un delito (86.3\%), el $41.2 \%$ admitió haber cometido este tipo de plagio durante su vida universitaria, y sólo el $11.8 \%$ afirmó haber recibido algún tipo de notificación por parte del docente cuando incurrió en plagio académico. En la parte final de este diagnóstico fueron proporcionados dos párrafos, extraídos del libro Thomas M. y Smith (2007), para que los estudiantes hicieran una paráfrasis de los mismos. Los resultados de esta sección coinciden con el poco conocimiento sobre paráfrasis, ya que únicamente el $3.9 \%$ de los estudiantes fueron capaces de realizar correctamente la paráfrasis del texto proporcionado y el 23 . $5 \%$ cometió plagio académico.

Figura 2

Resultados del diagnóstico

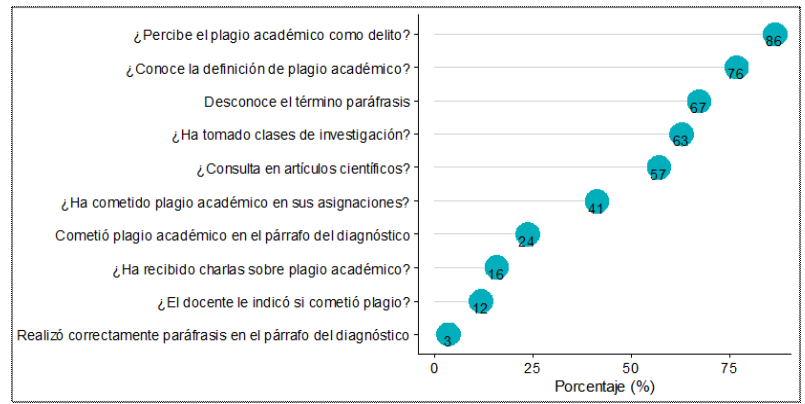

Nota: Resultados del diagnóstico aplicado a los estudiantes de los tres espacios pedagógicos. El eje vertical muestra algunas preguntas y aspectos consultados, en el eje horizontal se indican los porcentajes correspondientes. 
Etapa 1: fueron analizados 74 ensayos, 28 de los cuales corresponden a la Etapa 1 y el resto a la Etapa 2. Los resultados del ANOVA para la Etapa 1 no mostraron alguna diferencia significativa tanto para el criterio $1(F=1.005, p=0.38)$ como para el criterio $2(F=0.84, p=0.44)$ en los tres espacios pedagógicos. La uniformidad en los niveles de plagio, independiente del espacio pedagógico, era de esperarse, debido a que en esta etapa ningún estudiante recibió información en la prevención de plagio académico.

Etapa 2: el tipo de estrategia utilizada tiene un impacto significativo en la disminución de los niveles de plagio ( $C 1: F=9.039, p=0.000529$; C2: $F=4.878, p=0.0123$ ). Los niveles más bajos de plagio fueron registrados en los trabajos de los estudiantes que utilizaron el i_Map (test Tukey, $p=0.0003246$ ), de igual forma los valores medios más altos de fuentes de mayor crédito académico fueron registrados en el espacio pedagógico que utilizó el i_Map $(p=0.0100)$. No fueron registradas diferencias estadísticamente significativas entre los estudiantes del equipo control y el de los talleres de citas, como se observa en la Figura 3.

Figura 3

Éxito en prevención del plagio según el tipo de estrategia utilizada

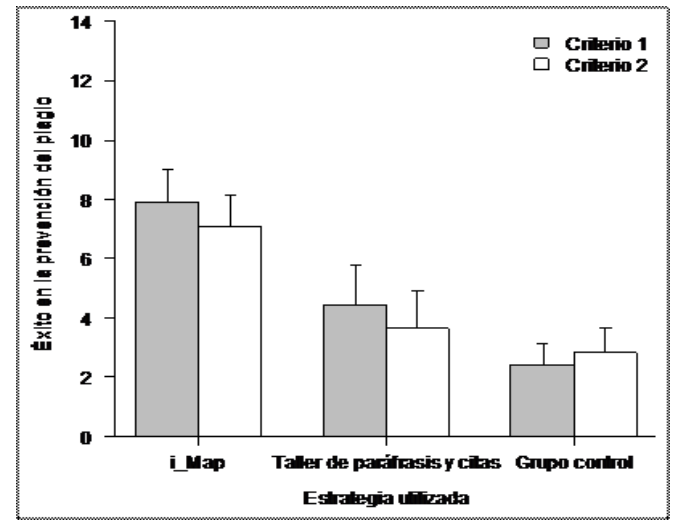

Nota: Las barras color gris hacen referencia al Criterio 1 y las barras sin relleno al Criterio 2. Las líneas verticales sobre cada barra representan el error estándar del conjunto de datos. $\mathrm{C} 1=$ cantidad de citas correctas (libres de plagio); $\mathrm{C} 2=$ número de fuentes de mayor crédito académico. 
Antes y después del uso del i_map

Las asignaciones de los estudiantes que utilizaron el i_map, mostraron avances significativos en la disminución de los niveles de plagio. El resultado del Welch test reveló que existe una diferencia significativa en los criterios 1 y 2 de los ensayos de la Etapa 1 y la Etapa 2 (C1: $t=$ -7.957, $d f=7.018, p$-value $=9.308 \mathrm{e}-05 ; \mathrm{C} 2: t=-6.8279, d f=6.814, p$ value $=0.0002785)$, develando la fuerte influencia del uso de esta estrategia en la prevención del plagio académico (Figura 4).

Figura 4

Avance de los estudiantes que utilizaron el i_map en la elaboración de los ensayos

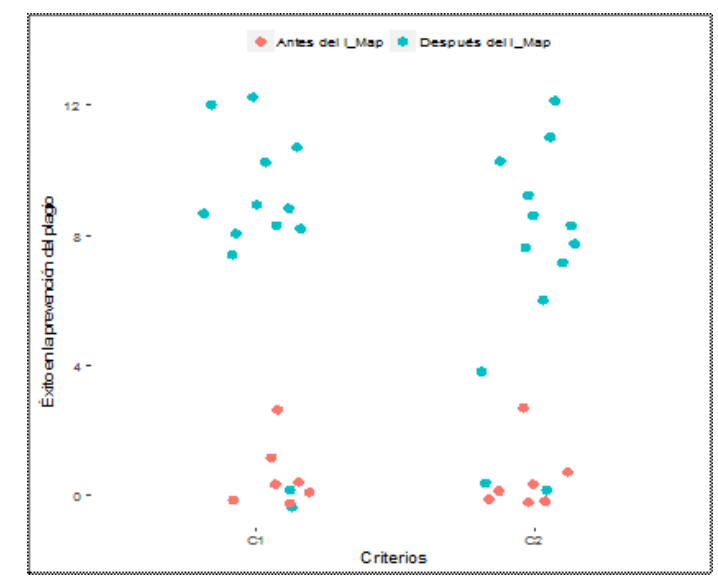

Nota: El gráfico muestra el comportamiento de los niveles de plagio registrado en las asignaciones de los estudiantes antes (puntos color rosa) y después (puntos color azul) del uso del i_map. Los puntos representan el éxito en la prevención del plagio medido a través de los Criterios 1 y $2(\mathrm{C} 1, \mathrm{C} 2)$. C1=cantidad de citas correctas (libres de plagio); C2= número de fuentes de mayor crédito académico.

\section{Conclusiones}

Los resultados de este estudio prueban la efectividad del i_map en la prevención del plagio académico. La forma en la que esta estrategia 
es útil en la disminución de los niveles de plagio es por su enfoque en el fortalecimiento de las habilidades de búsqueda y manejo de información (fases 1-3 del i_map), inducción del estudiante en la creación de una idea propia con base a la información colectada (fase 4 del i_map) y selección de ideas claves de otros autores que darán mayor fundamentación teórica a la idea generada (fase 5 del i_map). Específicamente, la fase 5 del i_map requiere que el estudiante reconozca que las ideas que incluirá en su trabajo corresponden a otros autores y que estás deberán tener el crédito correspondiente. Es decir, el i_map no está desligado de la enseñanza de las normativas de citas existentes, ya que en dicha fase se introduce al estudiante al uso de las ideas de otros autores, generando un espacio oportuno para que el docente introduzca la normativa que se utiliza, por ejemplo, normas APA. Como lo menciona Walden y Peacock (2008), el i_map, hace mayor énfasis en "el proceso antes que el producto" (p.133), induciendo a los estudiantes a invertir mayor tiempo en los procesos de lectura, análisis y selección de la información que correctamente deberán citar en sus trabajos, antes que en la creación de un texto sin enfatizar en dichos procesos. Los resultados del presente estudio indican que el plagio accidental puede ser reducido a través del i_map. Estudios previos que involucran el uso de esta estrategia también muestran resultados similares a los aquí registrados (Emerson, Stevens y Muirh, 2008).

Como ha sido establecido por otros autores (Emerson, Stevens y Muirh, 2008; Walden y Peacock, 2008), el i_map tiene múltiples beneficios pedagógicos para los estudiantes que van más allá de la prevención del plagio. Así, en este estudio, también fueron observados dichos beneficios en los ensayos de los estudiantes, como ser: mejoramiento en la coherencia de transición de un párrafo a otro, información delimitada y pertinente al tema planteado y mejora en la contextualización de la temática abordada. Estos resultados fueron observados en esta investigación, más no fueron medidos, por lo que sugerimos sean considerados en estudios futuros que tengan como fin ahondar o evaluar en el mejoramiento de otras habilidades o beneficios proporcionados por esta estrategia. 
El i_map mostró ser la estrategia más efectiva en la prevención del plagio en comparación a los talleres de paráfrasis y el equipo control. Como establecido por Holt (2012), los procesos educativos, particularmente el reforzamiento en las habilidades de paráfrasis, previenen el plagio; así era de esperar una diferencia estadísticamente significativa en los niveles de plagio de los estudiantes del equipo control y el de los talleres de paráfrasis y citas. Sin embargo, dicha diferencia no fue registrada entre estas dos estrategias, indicando que sólo el reforzamiento en paráfrasis y citas textuales puede no ser suficiente para prevenir el plagio académico. Algunas de las limitaciones de este estudio se enmarcan en la dificultad de dar seguimiento a cada uno de los participantes, debido a los retiros o abandonos de algunos estudiantes en los espacios pedagógicos. Se sugiere que estudios futuros sean desarrollados con espacios pedagógicos de mayor número de estudiantes.

Como fue establecido en los resultados, los estudiantes perciben el plagio académico como un delito, por lo tanto, es importante establecer por qué éste es un acto deshonesto y no únicamente condenarlo. A pesar de que los estudiantes tienen esta percepción y que algunos de ellos admitieron haber cometido plagio (41.2\%), únicamente un bajo porcentaje $(11.8 \%)$ afirmó recibir algún tipo de comunicación por parte del docente de haber incurrido en esta práctica. Lo anterior devela la necesidad de abordar y fortalecer estas temáticas no únicamente con los estudiantes, también con los docentes.

Con base a los i_map elaborados por los estudiantes, concluimos que es una herramienta útil no sólo para el estudiante, sino también para el docente, brindándole a éste una oportunidad para retroalimentar y reforzar las competencias de análisis, recolección y selección de información. Además, la prevención del plagio establece las bases para desarrollar el sentido de propiedad intelectual fortaleciendo las competencias de comunicación e investigación en los estudiantes. 
Efectividad del i_map en la prevención de plagio académico a nivel universitario

\section{Referencias Bibliográficas}

American Psychological Association. (2010). Publication Manual of the American Psychological Association. Washington, DC 20002: American Psychological Association.

Beasley, D. J. (2004). The Impact of Technology on Plagiarism Prevention and Detection:Research Process Automation, a New Approach for Prevention. Plagiarism: Prevention, Practice and Policies, 1-11.

Bennett, R. (2005). Factors associated with student plagiarism in a post-1992 university. Assessment \& Evaluation in Higher Education, 30:2, 137-162. Doi: 10.1080/0260293042000264244.

Berlinck, R. G. (2011). The academic plagiarism and its punishments a review. Brazilian Journal of Pharmacognosy, 365-372.

Centro de escritura Javeriano. (2011). Normas APA sexta edición. Cali: Pontificia Universidad Javeriana.

Diez-Martínez, E. (2014). Deshonestidad académica de alumnos y profesores. Su contribución en la desvinculación moral y corrupción social. Sinéctica. Revista electrónica de educación, 1-17. Obtenido de: h t t p: / / w w w. sin e c t ic a. it e so.m x/articulo / ?id=44_deshonestidad_academica_de_alumnos_y_profesores_su_ contribucion_en_la_desvinculacion_moral_y_corrupcion_social.

Emerson, L., Stevens, S., \& Muirhead, J. (2008). Information Maps: Supporting Students Through Their Research and Writing Processes. Good Practice Publication Grant scheme AKO AOTEAROA, 1-15.

Fintan, C., \& Lancaster, T. (2001). Plagiarism issues for higher education. VINE, 31, 36-41.doi: 10.1108/03055720010804005. 
Gulliver, J., \& Tyson, G. A. (2010). Exploring university students' perceptions of plagiarism: a focus group study. Studies in Higher Education, 35:4, 463-481. Doi: 10.1080/03075070903096508.

Holt, E. A. (2012). Education Improves Plagiarism Detection by Biology Undergraduates. Bio Science, 62:6, 585-592.Obtenido de: http:// www.bioone.org/doi/full/10.1525/bio.2012.62.6.9

Office of Science and Technology Policy. (2000, Diciembre 6). The Office of Research Integrity. Retrieved from https://ori.hhs.gov/federalresearch-misconduct-policy

Online Writing Lab at Purdue University. (2013, Febrero 15). Quoting, Paraphrasing, and Summarizing. Retrieved from https:// owl.english.purdue.edu/owl/resource/563/1/

Plagiarisma. (2010). Plagiarisma Duplicate Content Checker for Your Essay. Retrieved from http://plagiarisma.net/

Tomas M., S. M., \&Smith, R. L. (2007). Ecología. Madrid: PEARSON EDUCACIÓN, S.A. ISBN: 978-84-7829-084-0.

Plagiarism . (2017, Mayo 18). Plagiarism . Retrieved from ¿What is Plagiarism?: http://www.plagiarism.org/article/what-is-plagiarism

Voelker, T. A., Love, L. G., \& Pentina, I. (2011). Plagiarism: What Don't They Know? Journal of Education for Business (Walden \& Peacock, 2008), 87:1, 36-41. doi: 10.1080/08832323.2011.552536.

Walden, K., \& Peacock, A. (2006). The i_Map: a process-centered response to plagiarism. Assesment \& Evaluation in Higher Education, 31:2, 201-214. doi: 10.1080/02602930500262510.

Walden, K., \& Peacock, A. (2008). Economies of plagiarism: The I_map and issues of ownership in information gathering. En C. Eisner, \& M. Vicinus, Originatlity, imitation, and plagiaris / Teaching writing in the digital age (págs. 133 - 145). The University of Michigan Press. 
Efectividad del i_map en la prevención de plagio académico a nivel universitario

Walker, A. L. (2008). Preventing Unintentional Plagiarism: A Method for Strengthening Paraphrasing Skills. Journal of Instruccional Psychology, 35, 387-395.

<Paradigma> - Revista de Investigación Educativa. Año 24. No. 38 